\title{
Supercontinuum generation with microstructured fibers made of soft glass
}

\author{
Ryszard Buczynski ${ }^{1,2}$, Dariusz Pysz ${ }^{2}$, Ireneusz Kujawa ${ }^{2}$, Jakub Korzeniowski ${ }^{1}$, \\ Tadeusz Martynkien ${ }^{3}$, Francis Berghmans ${ }^{4}$, Hugo Thienpont ${ }^{4}$, and Ryszard Stepien ${ }^{2}$ \\ ${ }^{1}$ Faculty of Physics, University of Warsaw, Pasteura 7, 02-093 Warsaw, Poland, \\ ${ }^{2}$ Institute of Electronic Materials Technology, Wólczyńska 133, 01-919 Warsaw, Poland \\ ${ }^{3}$ Institute of Physics, Wroclaw University of Technology, Wybrzeze Wyspianskiego 27, Wroclaw, Poland \\ ${ }^{4}$ Dep. of Applied Physics and Photonics, Vrije Universiteit Brussel, Pleinlaan 2, Brussels, Belgium
}

Received September 28, 2009; accepted September 29, 2009; published September 30, 2009

\begin{abstract}
In this paper we report on the fabrication of a microstructured fiber made of in-house synthesized silicate glass, with a nonlinear Kerr refractive index of $4.010^{-15} \mathrm{~cm}^{2} / \mathrm{W}$. The fiber uses three rings of holes around a slightly elliptical core with dimensions $2.6 \mu \mathrm{m} x$ $3.4 \mu \mathrm{m}$. It has a birefringence of about $10^{-3}$ at $1.5 \mu \mathrm{m}$ and zero dispersion wavelengths at $860 \mathrm{~nm}$ and $870 \mathrm{~nm}$. Using this fiber we have demonstrated ultra broadband supercontinuum generation in the 400 $1600 \mathrm{~nm}$ range for a $19.5 \mathrm{~cm}$ sample pumped with 100 fs pulses with central wavelength of $755 \mathrm{~nm}$ and energy of $2 \mathrm{~nJ}$. The broadband generation of $200 \mathrm{~nJ}$ in the $650-850 \mathrm{~nm}$ range with pulse energy at the level of $0.57 \mathrm{~nJ}$ is also observed with the same structure.
\end{abstract}

Supercontinuum generation (SG) has been investigated since the mid 70 -ties ${ }^{1}$ however photonic crystal fibers (PCF) brought new opportunities to build novel types of supercontinuum sources with improved energy conversion efficiency and with low threshold power ${ }^{2}$. In our work we focus on the development of PCFs with soft glass since the nonlinear refractive index of such glass types can be considerably higher than in conventional silica glass. Moreover, the transmission window in soft glasses can permit the generation of supercontinuum in the Mid IR range up to 5 microns.

The microstructured photonic fiber labeled NL12 was fabricated with a borosilicate glass NC-21 synthesized inhouse at the Institute of Electronic Materials Technology (ITME). The material properties of this glass are: refractive index $\mathrm{n}_{\mathrm{D}}=1.518$, density $\rho=2.50 \mathrm{~g} / \mathrm{cm}^{3}$, coefficient of thermal expansion $\alpha_{20-300}=8210^{-7} \mathrm{~K}^{-1}$, glass transition temperature $\mathrm{T}_{\mathrm{g}}=500^{\circ} \mathrm{C}$ and dilatometric softening point $\mathrm{DTM}=545^{\circ} \mathrm{C}$. The nonlinear refractive index of silicate glass $\mathrm{NC} 21$ is similar to that of pure silica. Its main advantage is low temperature performance that allows preparing non-conventional complex fiber structures.

For the structure preform preparation we use glass tubes made of NC-21 glass with optimized thickness and calibration to avoid structure distortion. The fiber drawing relies on a standard stack and draw technique, but for the subpreform we use a low speed drawing process with modified parameters to ensure homogenous heat distribution in the subpreform and a lower temperature to preserve a high fill factor of the photonic lattice. Different fibers with regular hexagonal lattice and small core in the center have been obtained (Fig. 1).
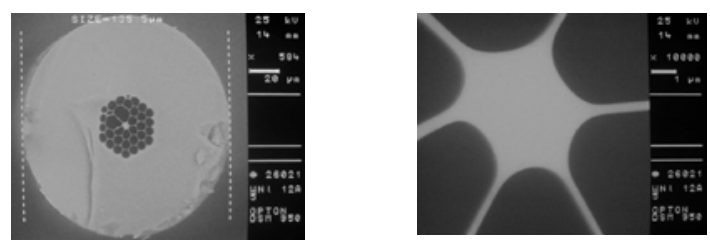

Fig.1. Scanning Electron Micrographs of the small core PCF NL12 fabricated with borosilicate glass NC-21.

The non-uniformity of the photonic cladding leads to an elliptical core with the dimensions of about 3.4 and 2.6 $\mu \mathrm{m}$ along the main axes. The fibres are therefore birefringent in the fundamental mode. Most of these fibers also exhibited unintentional cracking of the glass matrix. We suppose that this results from inadequate handling during the preform assembly.

The modal and dispersion properties of the PCF NL12A5 are calculated by a full vectorial biorthonormal basis method $(\mathrm{BBM})^{3}$. This method uses a matrix representation of the vector wave equations calculated in the base of plane waves and solves an eigenvalue problem for that matrix. The method allows predicting modal, polarization and dispersion properties of a PCF with periodic boundary conditions. The linear modal properties of the fiber are calculated based on the actual real structure obtained from scanning electron micrographs (Fig.1). The simulation therefore takes into account all the deformations of the structure. In the modeling we also take into account the dispersion of bulk NC-21 glass through the Sellmeier coefficients ${ }^{4}$. According to the modeling results the fabricated fiber has a birefringence on the level of $10^{-3}$ at $1.5 \mu \mathrm{m}$ (Fig.2). Zero 
dispersion of fundamental mode is achieved for the wavelengths of $860 \mathrm{~nm}$ and $870 \mathrm{~nm}$, respectively (Fig.3). However, also few higher modes propagates in the fiber and we suppose that they also can take part in supercontinuum generation. For the experiment we used a tunable ultrafast Ti:Sapphire oscillator Tsunami Millennia Pro 10 of Spectra Physics, which delivers pulses with a constant length of $100 \mathrm{fs}$ and an average output optical power up to $1 \mathrm{~W}$ (depending on the wavelength).
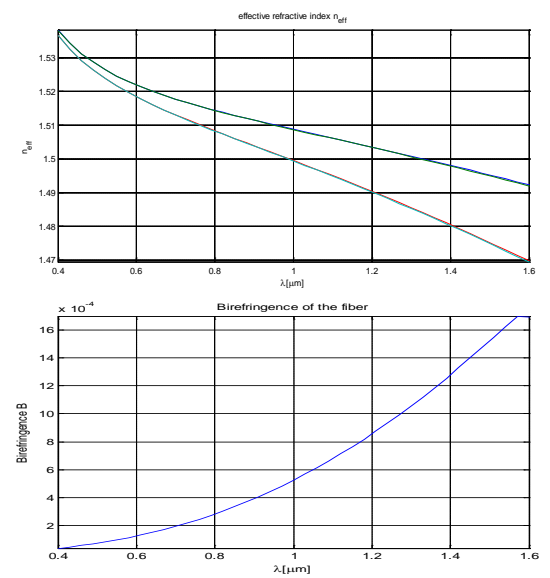

Fig.2. Calculated effective index of guided modes and phase birefringence of PCF NL12 calculated with BBM.



Fig.3. Calculated dispersion properties of PCF NL12

Taking into account the repetition rate of the oscillator of $80 \mathrm{MHz}$, the maximum peak power is equal to $125 \mathrm{~kW}$ while the maximum energy in the pulse reaches $12.5 \mathrm{~nJ}$. We couple light into the PCF with a microscope objective with X 40 magnification. We measured coupling efficiency to be around $30 \%$ depending on the fiber sample. The output radiation was coupled into an Instrument Systems spectrometer to record spectra in the 400-800 nm range and into an ANDO optical spectrum analyser in the visible range and in the near IR (600-1750 $\mathrm{nm})$. We have examined the fiber sample with a $19.5 \mathrm{~cm}$ length. The growth of the generated spectrum as a function of pulse energy is shown in the visible (Fig.4) and in the near IR regions (Fig.5). Broadening of the spectrum in the visible region is observed with a pulse energy as low as $0.57 \mathrm{~nJ}$. In this case a strong peak at 490 $\mathrm{nm}$ appears. Increasing the peak energy up to $1.14 \mathrm{~nJ}$ broadens the spectrum and new peaks appear around 540 and $650 \mathrm{~nm}$. In the near IR we observe the generation of solitons that shift towards longer wavelengths when pulse energy increases. With a further energy increase we observe soliton fission. Beside the Kerr nonlinearity driven soliton formation process the stimulated Raman scattering (SRS) is the second important phenomena responsible for spectral broadening. In the near IR we observe the generation of solitons that shift towards longer wavelengths when pulse energy increases. Beside the Kerr nonlinearity driven soliton formation process the stimulated Raman scattering (SRS) is the second important phenomena responsible for spectral broadening. The generation of the spectrum in the visible range can be explained by the generation of dispersive waves related to solitons shifting to the red.

a)
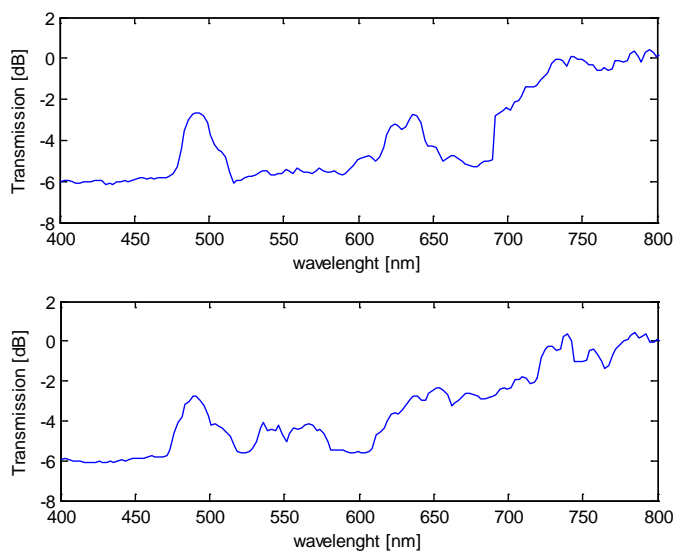

b)

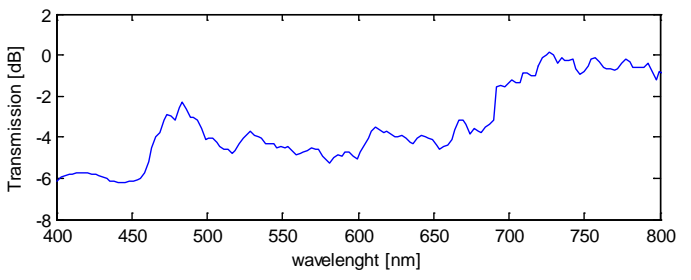

c)

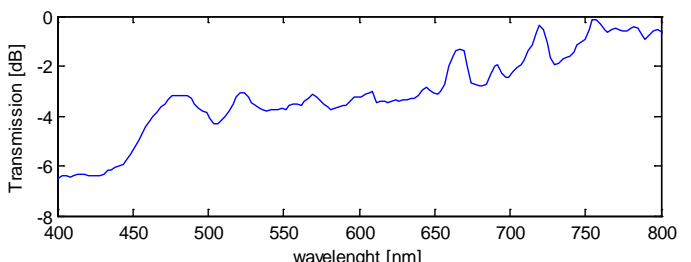

d)

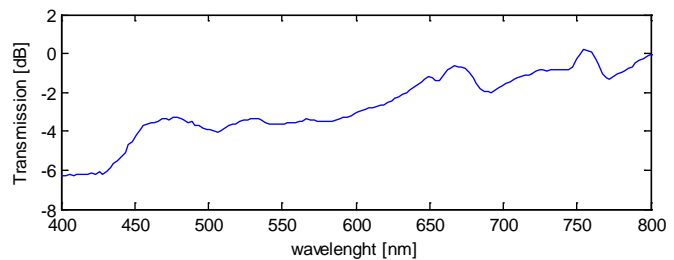

Fig.4. Supercontinuum generation for pulses of $100 \mathrm{fs}$, central wavelength $755 \mathrm{~nm}$ and pulse energy: $0.57 \mathrm{~nJ}$ (a), $0.75 \mathrm{~nJ}$ (b), $1.14 \mathrm{~nJ}$ (c), $1.92 \mathrm{~nJ}(\mathrm{~d}), 2.78 \mathrm{~nJ}$ (e). The spectra are recorded in the 400-800 $\mathrm{nm}$ range. The fiber length is $19.5 \mathrm{~cm}$

We can observe this if the generated peaks move towards the blue with increasing pulse energy. When the 
pulse energy increases up to $2 \mathrm{~nJ}$, we can observe continuous spectra in the 400-1600 nm range (Fig.4d, Fig.5d, Fig.6). A further increase of pulse energy up to 4 $\mathrm{nJ}$ causes the spectra to smoothen, but it does not influence the broadening of the spectra itself (Fig.4e, Fig.5e, 5f).

(a)
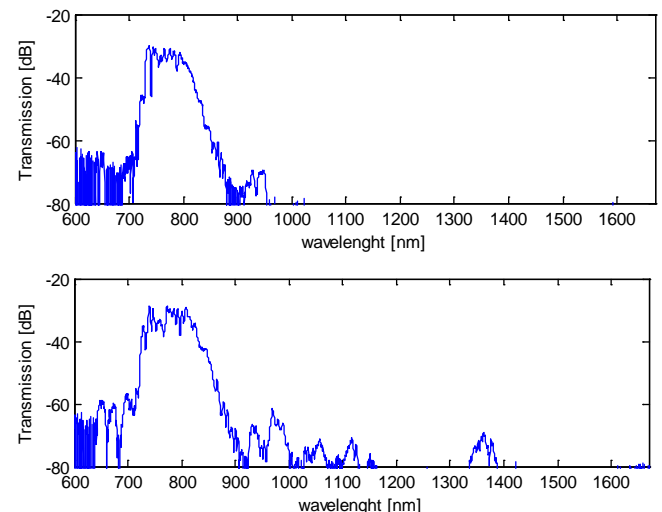

(b)

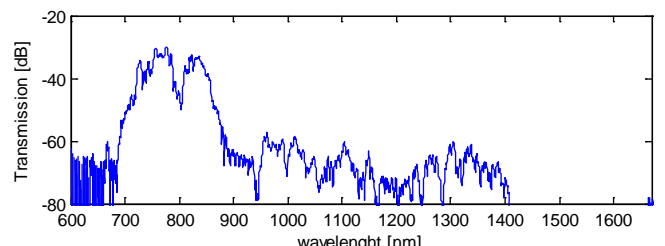

(c)

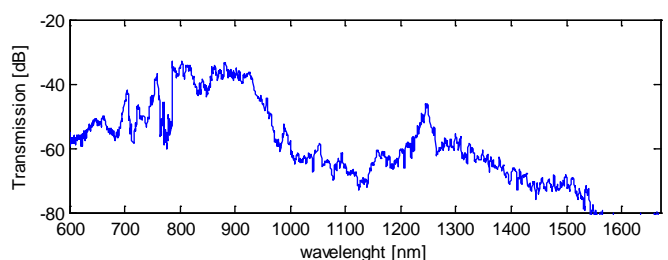

(d)

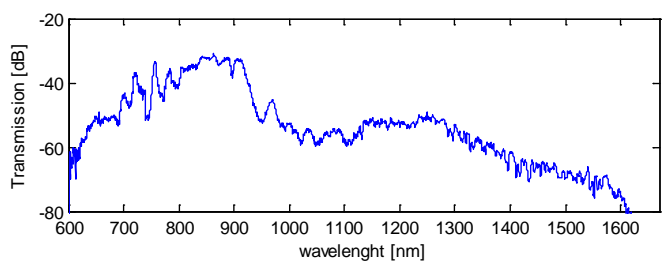

Fig.5. Supercontinuum generation for pulses of $100 \mathrm{fs}$, central wavelength $755 \mathrm{~nm}$ and pulse energy: $0.57 \mathrm{~nJ}(\mathrm{a}), 0.75 \mathrm{~nJ}(\mathrm{~b}), 1.14 \mathrm{~nJ}(\mathrm{c})$, $1.92 \mathrm{~nJ}(\mathrm{~d}), 2.78 \mathrm{~nJ}$ (e). Spectra are recorded in the range $600-1750 \mathrm{~nm}$. The fiber length is $19.5 \mathrm{~cm}$.

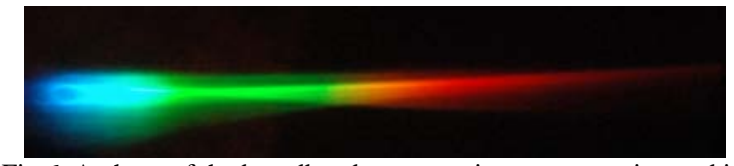

Fig.6. A photo of the broadband supercontinuum generation and its spectrum .

The features of the spectrum generated when the PCF is pumped with $755 \mathrm{~nm}$ prove the abnormal regime of operation. In the near IR we observe the generation of solitons that shift towards longer wavelengths when pulse energy increases. Beside the Kerr nonlinearity driven soliton formation process the stimulated Raman scattering (SRS) is the second important phenomena that might be responsible for spectral broadening in the IR region.

To identify the optimum wavelength for fiber excitation we tuned the central wavelength from $755 \mathrm{~nm}$ towards longer wavelengths. With an increasing wavelength the spectrum in the visible range decreases and disappears in the blue region (Fig.7). The spectrum is no longer continuous in the visible region when the pump pulse reaches the central wavelength of $815 \mathrm{~nm}$. This phenomenon might be related to the shift of the pump wavelength from the abnormal to the normal dispersion regime. This does not match with the simulation results that predicted zero dispersion in the $860 \mathrm{~nm}$ region. The reason for this difference is probably to be found in the low accuracy of the simulations (an increase of plane waves in the base is needed), uncertainty in the Sellmeier coefficient calculation for NC-21 glass and/or inaccurate dimensions obtained from the scale readings on the scanning electron micrograph.



Fig.7. Supercontinuum generation for pulses of $100 \mathrm{fs}$, pulse energy $1.92 \mathrm{~nJ}$, pump wavelength $815 \mathrm{~nm}$.

Ultra broadband supercontinuum generation in the range 400-1600 $\mathrm{nm}$ has been achieved with a $19.5 \mathrm{~cm}$ soft glass microstructured fibre sample pumped with 100 fs pulses with a central wavelength of $755 \mathrm{~nm}$ and energy of $2 \mathrm{~nJ}$. The broadband generation of $200 \mathrm{~nm}$ in the $650-850 \mathrm{~nm}$ range is observed with pulse energy at the level of $0.57 \mathrm{~nJ}$.

This work is supported by the Polish Ministry of Science research grant R0204302, by the COST 299 action, by the EU FP6 Network of Excellence on MicroOptics "NEMO" and by an internal scientific grant of ITME. Numerical results were partially obtained using computer resources of the Interdisciplinary Centre for Mathematical and Computational Modelling (ICM) at the University of Warsaw.

\section{References}

[1] R. Alfano, The Supercontinuum Laser Source: Fundamentals with Updated References, (Springer, 2005).

[2] J. M. Dudley et al., Rev. Mod. Phys. 78. 1135-1184, (2006)

[3] A.Ferrando et al., Opt. Lett. 24, 276 (1999)

[4] I.Kujawa et al., Proc. SPIE Vol. 6182, 61822Q (2006) 